Salazar-Jiménez, R. (2019). Evaluación de la implementación de materiales didácticos en Ciencias Sociales: propuestas para el desarrollo de la competencia social y ciudadana. Revista Electrónica Interuniversitaria de Formación del Profesorado, 22(2), 111-122.

\title{
Evaluación de la implementación de materiales didácticos en Ciencias Sociales: propuestas para el desarrollo de la competencia social y ciudadana
}

Rodrigo Salazar-Jiménez (Universidad del Bío-Bío, Chile)

\section{Resumen}

La información que se presenta en este artículo $^{1}$ se centra en la evaluación de la implementación de cinco propuestas didácticas en Ciencias Sociales, para el desarrollo de la competencia Social y Ciudadana, particularmente en la percepción del profesorado sobre el material propuesto. Los resultados se obtuvieron por medio de encuestas que los y las docentes de nueve institutos de Cataluña debían responder antes de aplicar las unidades didácticas y después de cada una de las sesiones.

El procesamiento de la información se realizó con un modelo de análisis cuantitativo con el uso del programa Excel. Entre los principales resultados que podemos destacar de la información entregada por los nueves docentes que aplicaron el material: el profesorado hace una evaluación positiva de las unidades didácticas; las actividades son motivadoras para el alumnado; por último, que para los y las docentes el material resulta útil para conseguir los objetivos de aprendizaje propuestos al alumnado.

\section{Palabras clave}

Formación ciudadana; materiales didácticos; rol docente; ciudadanía

\section{Contacto:}

Rodrigo Salazar-Jiménez, rsalazar@ubiobio.cl, Chillán: Avda. Andrés Bello 720, Casilla 447 - CP: 3800708.

${ }^{1} \mathrm{El}$ presente artículo recoge en parte los resultados obtenidos de la investigación titulada: "Desarrollo de la formación sociopolítica para una ciudadanía democrática: diseño e implementación de materiales didácticos en Ciencias Sociales (I+D EDU 2015-65621-C3-3-R)" realizada por el grupo DHIGECS de investigación en Didáctica de las Ciencias Sociales de la Universitat de Barcelona, siendo el Dr. J. Prats el investigador principal. Dicho proyecto se centra en el análisis de los mecanismos que ayudan a construir las identidades y la competencia social en los estudiantes de Educación Secundaria Obligatoria a través de contenidos históricos. 


\title{
Evaluation of the implementation of didactic materials in Social Sciences: proposals for the development of social and civic competence
}

\begin{abstract}
The information presented in this article focuses on the evaluation of the implementation of five didactic proposals in Social Sciences, for the development of Social and Citizen competence, particularly in the perception of the teaching staff about the proposed material. The results were obtained through surveys that the teachers of nine institutes in Catalonia had to answer before applying the teaching units and after each of the sessions.

The processing of the information was done with a quantitative analysis model with the use of the Excel program. Among the main results we can highlight the information provided by the nine teachers who applied the material: the teachers make a positive evaluation of the teaching units; the activities are motivating for the students; finally, that for teachers the material is useful to achieve the learning objectives proposed to the students.
\end{abstract}

\section{Key words}

Citizen education; didactic materials; teaching role; citizenship.

\section{Introducción}

Este artículo se enmarca en un proyecto del Grupo de investigación DHIGECS (Didáctica de la Historia, Geografía y otras Ciencias Sociales) titulado «Desarrollo de la formación sociopolítica para una ciudadanía democrática: diseño e implementación de materiales didácticos en Ciencias Sociales» (EDU2015-65621-C3-3-R). A continuación, se presenta la evaluación realizada por los docentes que aplicaron las cuatro de las experiencias de enseñanza-aprendizaje.

El grupo de investigación DHIGECS tiene experiencia en la elaboración y evaluación de prototipos didácticos (Prats, Molina-Neira, Ruíz y Molina, 2017), entre los que destacan:

- Programa educativo Aprenem a votar: Eleccions al Parlament de Catalunya 2010 (Prats y Wilson, 2013).

- El Aula como Laboratorio Histórico: La Guerra Civil Española 2014 (Salazar-Jiménez, 2016; Salazar-Jiménez, Orellana-Fonseca, Muñoz-Labraña y Bellatti, 2017)

- El Nacimiento del Facsismo 2014 (Salazar-Jiménez, Barriga-Ubed y Ametller-López, 2015; Salazar-Jiménez, 2016)

- Programa educativo Aprender a votar con las elecciones al Parlamento Europeo 2014 (Barriga-Ubed, Wilson-Daily y Prats-Cuevas, 2017)

Estos conocimientos sobre implementación y evaluación de prototipos didácticos y las diversas publicaciones sobre el tema, han dado una base sólida al grupo de investigación e innovación DHIGECS sobre formación ciudadana y educación cívica. Esto ha permitido abrir una línea de investigación y elaboración de materiales educativos relacionadas con la formación sociopolítica. Las propuestas didácticas elaboradas por el equipo para esta investigación son (Prats, Fuentes y Sabariego, 2019): 
- Mujeres y Revolución Industrial: Esta unidad didáctica acerca la primera Revolución Industrial desde una perspectiva de género a los estudiantes de $4^{\circ}$ de Enseñanza Secundaria. Partiendo del análisis de fuentes y de la búsqueda histórica, el estudiante se plantea preguntas iniciales que debe resolver con la reflexión y el análisis como actividades cognitivas fundamentales, emulando el trabajo del historiador.

- Atenas del origen de la democracia: Esta propuesta está pensada para ser aplicada en los primeros cursos de la educación secundaria e ilustra sobre las diferentes formas de participación social ciudadana, realizando un viaje que se inicia en la democracia ateniense y finaliza en nuestro sistema representativo actual, y haciendo especial hincapié en el tratamiento transversal del concepto de ciudadanía y participación política.

- Los amores de mi vida: Se propone como una secuencia didáctica transversal e interdisciplinar que tiene como objetivo reflexionar sobre el concepto del amor en su acepción más amplia. En este sentido, se persigue convertirse en un instrumento de prevención de actitudes de violencia de género, potenciando las relaciones basadas en el respeto y la igualdad.

- The Maze: Esta propuesta pretende el uso de la gamificación como base metodológica para el estudio del pasado. Partiendo del diseño de un juego de rol, se insta al estudiante a reflexionar sobre aspectos relacionados con la construcción de las identidades; los derechos humanos; la democracia y los totalitarismos entre otros.

Los resultados de la evaluación se presentan desde tres perspectivas: i) características de los participantes en cada una de las Propuestas Didácticas, donde se ha puesto énfasis en los datos sobre el centro y el grupo donde se aplica.; ii) se presentan los resultados de la evaluación de los docentes a la aplicación de los Materiales Didácticos; iii) por último, se presentan algunas opiniones de los/as docentes sobre las actividades por sesión.

\section{Fundamentación teórica}

Entre los objetivos de la enseñanza de las Ciencias Sociales es formar ciudadanos críticos, en un proceso de enseñanza-aprendizaje que abarque las distintas etapas de la vida escolar (Prats, 1997; Molina-Neira, 2017). En este sentido, la Historia y las Ciencias Sociales, permiten comprender desde las bases los problemas de actualidad y las claves del funcionamiento social (Prats y Santacana, 2011).

Resulta interesante que la reflexión sobre el ciudadano o ciudadana se quiere formar y para qué, se dé desde la didáctica de las ciencias sociales, contemplando las limitaciones y el potencial cognitivo de los jóvenes en sus diferentes etapas (Molina-Neira, 2017).

A continuación, se desarrollan las premisas teóricas sobre las que se llevó a cabo el análisis de resultados, así como los elementos referenciales que se consideran necesarios para llevar a cabo una interpretación de éstos.

La clasificación teórica sobre la ciudadanía resulta relevante para caracterizar la diversidad de posiciones de los diferentes agentes respecto a la Formación Ciudadana, como apunta Redón (2010), un ejemplo, en el contexto de una sociedad neoliberal, el proyecto biopolítico de producción de la vida, ya no es del Estado, si no del Mercado, que difunde su dominio a través de todos los ámbitos de la sociedad; por tanto, el Estado pasa a ser una agente más del proyecto neoliberal, tal como la escuela. 
Actualmente la literatura científica tiende a dicotomizar la concepción de la Formación Ciudadana, desde la perspectiva curricular y didáctico, se reconoce el enfoque minimalista orientado a la educación cívica tradicional y el maximalista, dirigido al desarrollo de competencias ligadas a la ciudadanía (Kerr, 2002; en Reyes, Campos, Osandón y Muñoz, 2013).

Continuando con la idea anterior, la Formación Ciudadana, cumple la función de entregar conocimientos, habilidades y actitudes requeridas para la vida e institucionalidad democráticas. En este contexto, desarrolla temas vinculados a la historia política cercana como derechos humanos, autoritarismo, democracia, manejo crítico de la información, responsabilidad social, entre otros (Torres y Muñoz, 2014).

La Formación Ciudadana es visibilizada como una necesidad, ya que supone, por un lado, una forma de subsanar una carencia formativa en el plano de lo político, ya sea a causa de un entorno familiar y/o cercano donde no se ha dado una socialización política significativa, como de una formación política deficiente en el ámbito escolar (Orellana, Bivort, Martinez, Farias, Cárcamo, Blanco \& Perez, 2015). Desde aquí entonces se visibiliza la escuela como un ente educador y formador, en donde se constituyen los conocimientos compartidos y elaborados socialmente donde los niños, niñas y adolescentes le dan sentido a las prácticas cotidianas en su contexto social, es así como la escuela plantea, como intención propia, constituir los espacios y organizar los tiempos en que tiene lugar la primera experiencia común a toda la sociedad (Cerda, Magendzo, Santa y Varas, 2004).

La Formación Ciudadana puede contribuir, decisivamente o no, al desarrollo de capacidades intelectuales y morales que son fundamento de la capacidad de acción ciudadana de la sociedad. Es decir, de reflexión y acción sobre sí misma: sus problemas más apremiantes, sus fines, y los significados que la cohesionan. En este contexto, se destacan las seis prácticas educativas que propone la Civic Mission of Schools (Middaugh y Kahne, 2014; Barriga-Ubed y Salazar-Jiménez, 2015): Formación en el aula; actividades de aprendizajeservicio; actividades extraescolares; gobierno escolar; discusión de temas controvertidos; y, por último, simulacro de procesos democráticos.

Por su parte, González-Valencia y Santisteban-Fernández (2016) sostienen que las formas en que la formación ciudadana se concibe y materializa en el currículum y las prácticas pedagógicas, responden a tipologías muy variadas, pues la formación ciudadana es altamente contingente en términos sociales. El debate que genera desborda los campos pedagógicos, lo que significa el surgimiento de múltiples planteamientos, en relación con las diferentes posturas sobre el tema.

Gran parte del profesorado, sigue orientando el proceso de enseñanza-aprendizaje en los contenidos (Salazar-Jiménez, 2016), no se observan los cambios que implica la educación centrada en el desarrollo de habilidades y competencias (Gimeno, 2009; Molina-Neira, 2016), donde el proceso de enseñanza-aprendizaje se centra en el alumnado. El cómo se lleve la Formación Ciudadana al aula dependerá de la postura del o la docente (Castillo, 2016).

Para este reporte de investigación, es importante destacar el rol de la Formación en el Aula propuesto por los autores (Middaugh y Kahne, 2014; Barriga-Ubed y Salazar-Jiménez, 2015), donde el proceso de enseñanza-aprendizaje, se centra en el enfoque minimalista, entregando conocimiento sobre el Estado, la Historia, los derechos y la democracia. En esta línea, los temas controversiales, que, según Toledo, Magendzo, Gutiérrez e Iglesias (2015, p. 289) ... tienen relación directa con la violación a los Derechos Humanos de las personas.. en este sentido, los procesos didácticos para tratar temas controversiales se deben centrar en el conflicto y fundarse en la deliberación y el diálogo (Magendzo y Toledo, 2015). Por último, 
los simulacros de procesos democráticos, permiten al alumnado recrear procesos y procedimientos democráticos, como las elecciones (Prats y Wilson, 2013; Barriga-Ubed, Wilson-Daily y Prats-Cuevas, 2017).

\section{Metodología}

La metodología utilizada es de carácter cuantitativo y el alcance del estudio es descriptivo. Se utilizaron dos encuestas, la primera con preguntas abiertas previa a la aplicación de los materiales y la segunda a partir de la escala tipo Likert de cinco y diez punto y otras abiertas (Bizcarra y Pérez, 2015), con el fin de indagar sobre la aplicación de los materiales educativos en cada una de las sesiones.

El cuestionario se realizó en base a un "cuadro de dimensiones" (Tabla 1.), donde en primera instancia y por medio del tema de investigación, se generaron las dimensiones que se van a abordar, Características del grupo curso; y, en segundo lugar, una encuesta para observar la aplicación del material.

Posterior a la aplicación del cuestionario a los respectivos informantes, se realizó una base de datos a partir del Excel, donde se incluyeron las preguntas del instrumento con sus respuestas, para luego depurar la información y finalmente analizarlos.

Tabla 1.

Dimensiones

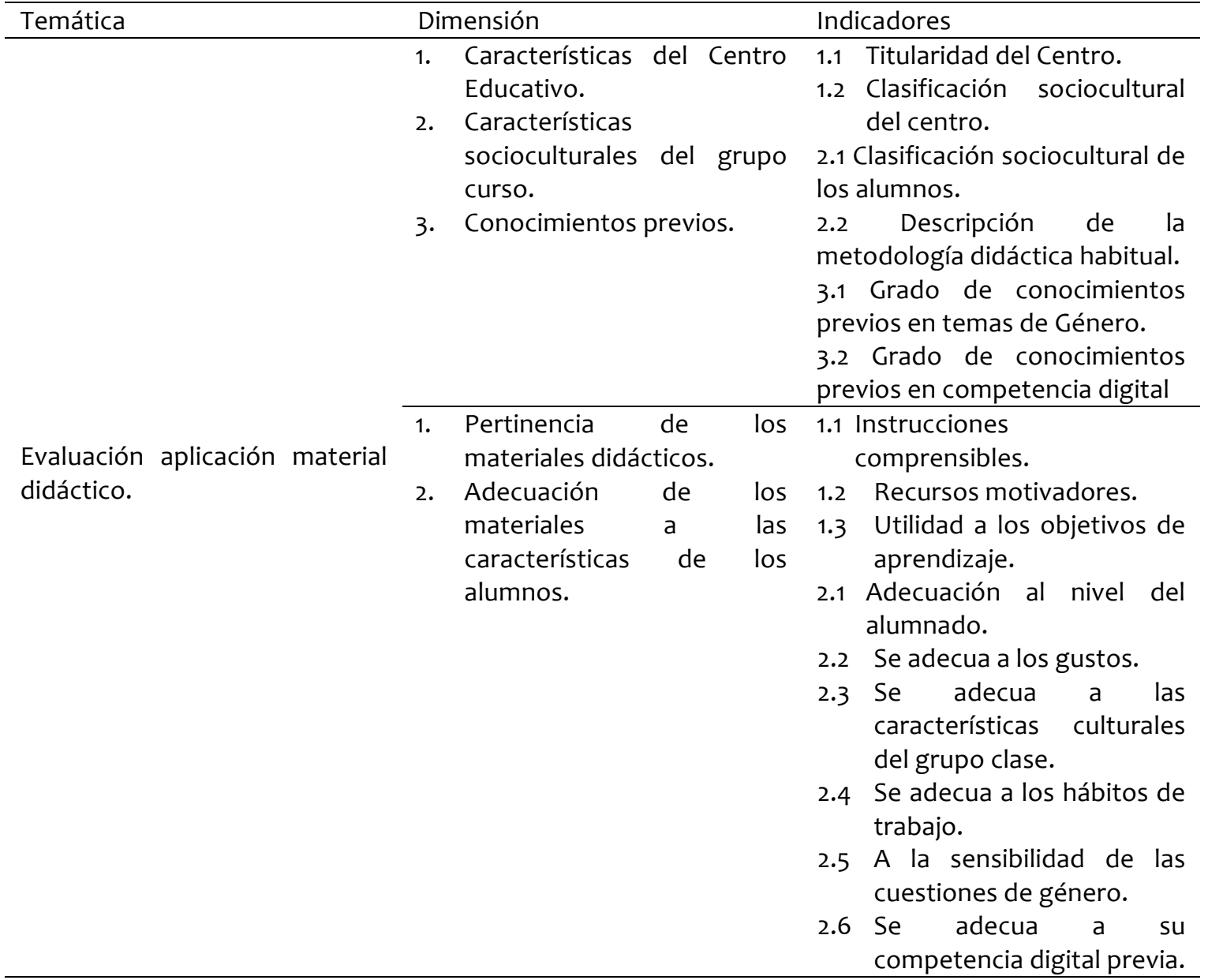




\section{Resultados}

Los resultados se presentarán, a partir de dos encuestas aplicadas a los docentes que aplicaron los materiales didácticos. La primera tiene relación con la descripción del centro educativo y el grupo donde se aplicó el material didáctico. La segunda encuesta los docentes la completaban al final de cada sesión y entregaban información sobre la aplicación del material.

Los materiales didácticos fueron aplicados $3^{\circ}$ de ESO, $4^{\circ}$ de ESO y $1^{\circ}$ de Bachillerato, durante el período escolar 2017-2018. Para implementar las propuestas didácticas se trabajó colaborativamente con doce docentes de Institutos en Cataluña. En total, el 45,5\% de los materiales didácticos se implementó en $3^{\circ}$ de ESO, el $45,5 \%$ en $4^{\circ}$ de ESO y un $9 \%$ en $1^{\circ}$ de bachillerato.

Tabla 2.

Cursos de aplicación

\begin{tabular}{lll}
\hline Docente & CursO & Propuesta Didáctica \\
\hline 1 & $4^{\circ} \mathrm{ESO}$ & Dones i revolució industrial \\
\hline 2 & $3^{\circ} \mathrm{ESO}$ & Els amors de la meva vida \\
\hline 3 & $3^{\circ} \mathrm{ESO}$ & Dones i revolució industrial \\
\hline 4 & $4^{\circ} \mathrm{ESO}$ & The Maze: Nosotros y ellos \\
\hline 5 & $4^{\circ} \mathrm{ESO}$ & Els amors de la meva vida \\
\hline 6 & $3^{\circ} \mathrm{ESO}$ & Dones i revolució industrial \\
\hline 7 & $4^{\circ} \mathrm{ESO}$ & The Maze: Nosotros y ellos \\
\hline 8 & $1^{\circ} \mathrm{Bachillerato}$ & The Maze: Nosotros y ellos \\
\hline 9 & $3^{\circ} \mathrm{ES}=$ & Els amors de la meva vida \\
\hline 10 & $4^{\circ} \mathrm{ESO}$ & Els amors de la meva vida \\
\hline 12 & $3^{\circ} \mathrm{ESO}$ & $\begin{array}{l}\text { Atenes: } L^{\prime} \text { origen de la } \\
\text { democràcia }\end{array}$ \\
\hline & &
\end{tabular}

En primer lugar, se presenta las características de los grupos a los que se implementaron los materiales didácticos. En total participaron 476 alumnos/as, promediando 33,5 por sesión. Según la información proporcionada por el profesorado los/as estudiantes que utilizó el material didáctico pertenece a un nivel sociocultural medio bajo. El alumnado que participo es de origen diverso, según lo reportado por los docentes, provienen de América del Sur, países Árabes, Ucrania y Cataluña.

Al consultar a los docentes sobre las metodologías didácticas que normalmente utilizan, estos señalan que trabajan generalmente por proyectos, trabajo en grupos cooperativos, con explicaciones teóricas, trabajo en soporte informático, etc.

Sobre conocimientos de la competencia digital, como se observa en la Figura 1, el profesorado que respondió la primera encuesta señala que el $45 \%$ de los cursos que participaron en la implementación de los materiales didácticos tiene un conocimiento Medio-Alto, el $45 \%$ un conocimiento Medio y el $11 \%$ solo alcanza un nivel de conocimiento básico. 


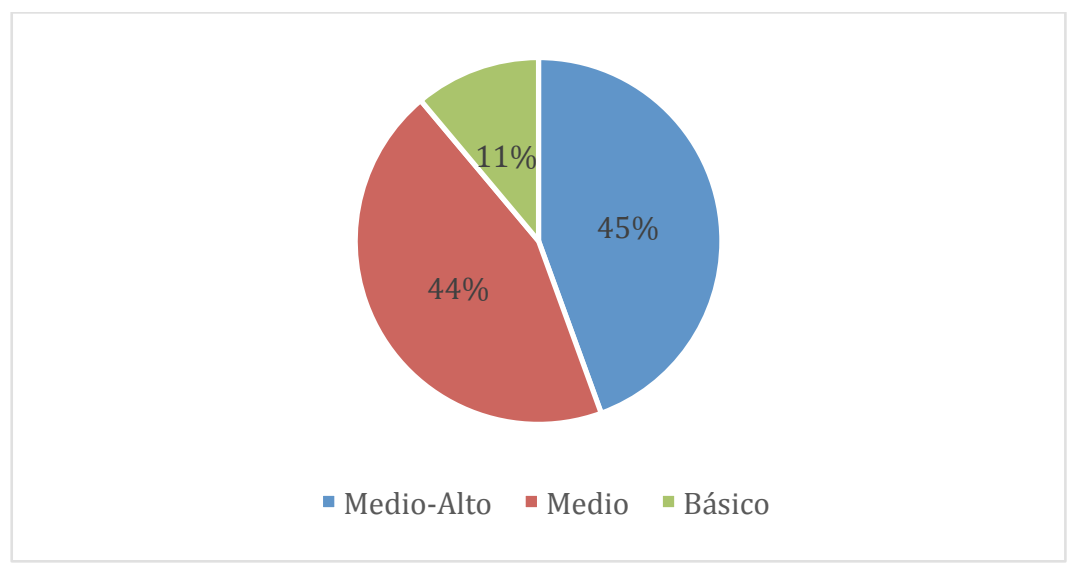

Figura 1. Conocimiento Digital.

Todos los docentes encuestados (Figura 2), señalan que los alumnos tienen conocimiento sobre temas de Género, pero solo un $38 \%$ dice que tienen un nivel de conocimiento alto y el $62 \%$ restante explica que tienen un nivel balo. Aquellos docentes que dicen que sus alumnos tienen un bueno conocimiento sobre temas relacionado con el género, destacan el compromiso del centro en estas cuestiones, como se explica a continuación:

Tenen coneixements previs en qüestions de gènere per: tallers previs. Enfocament transversal des de la matèria de ciències socials. Algunes noies participen voluntàriament en un grup feminista del centre.

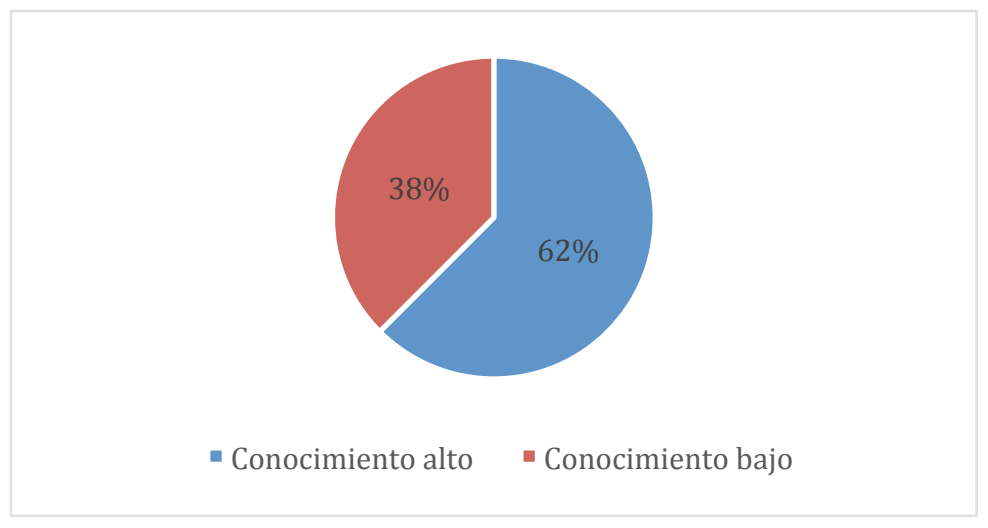

Figura 2. Conocimiento sobre temas de Género

En segundo lugar, se presenta la evaluación realizada por el profesorado a la aplicación de los materiales didácticos.

Como se observa en la Tabla 3 los/as docentes participantes destacan:

- El $86,9 \%$ de las actividades del material didáctico presenta instrucciones comprensibles para las/os estudiantes y solo en un 3,9\% de las sesiones las instrucciones serían poco comprensibles. 
- Según el profesorado que aplico el material didáctico, el 82,9\% de las actividades propuestas en cada una de sesiones han resultado motivadoras para los/as estudiantes.

- El 96,1\% del profesorado, señalo que el material didáctico les ha resultado útil para alcanzar los objetivos de aprendizaje en cada sesión.

Tabla 3

Evaluación de las sesiones

\begin{tabular}{lccccc}
\hline Indicadores & $\begin{array}{c}1 \\
\text { (Muy } \\
\text { poco) }\end{array}$ & 2 & 3 & 4 & $\begin{array}{c}5 \\
\text { (Mucho) }\end{array}$ \\
\hline Les instruccions són intel-ligibles per a l'alumnat? & $1,3 \%$ & $2,6 \%$ & $9,2 \%$ & $48,7 \%$ & $38,2 \%$ \\
\hline Ha resultat una sessió motivadora per a l'alumnat? & 0 & 0 & $17,1 \%$ & $38,2 \%$ & $44,7 \%$ \\
\hline $\begin{array}{l}\text { Creus que ha resultat útil per assolir els objectius } \\
\text { d'aprenentatge proposats a l'alumnat? }\end{array}$ & 0 & 0 & $3,9 \%$ & $46,1 \%$ & $50 \%$ \\
\hline
\end{tabular}

Al consultar a los docentes sobre si las sesiones se adecuaban a las características del alumnado, como se observa en la Tabla 4, señalan:

- Los/as docentes expresan que el $1,3 \%$ de las sesiones no se adecua al nivel académico de los/as estudiantes, el $11,9 \%$ de las sesiones algo y un $86,7 \%$ de las sesiones se encuentran adecuadas al nivel académico del alumnado.

- El $65 \%$ de las sesiones eran del gusto de los/as estudiantes y el $26 \%$ que era algo del gusto del alumnado.

- Según los/as profesores/as el 75\% de las sesiones se adecuaron a las características culturales de la clase y $25 \%$ se adecuaron solo algo.

- El $71,5 \%$ de las sesiones se adecuan a los hábitos de trabajo del alumnado y el $26 \%$ de los encuestados expresan que $26 \%$ de las sesiones algo se adecuo a los hábitos de trabajo.

- Según los docentes encuestados el 7,5\% de las sesiones no sensibilizaron sobre cuestiones de género, el $20,8 \%$ dice que algo y el $70,2 \%$ señala que las sesiones sensibilizan sobre cuestiones de género.

- Según los/as encuestados/as, el 12,7\% de las sesiones de adecuan algo a las competencias digital previa del alumnado y $83,2 \%$ se adecuan a las competencias digital de los discentes.

Entre las dificultades de la aplicación de los materiales didácticos, un docente que utilizo el prototipo titulado Mujeres y Revolución Industrial, en la segunda sesión señalo, ... les sessions d' exposició oral per grups es fan llargues, però donat el nombre de grups, s' ha tingut que allargar fora d' horari les exposicions.

Una docente que trabajo con la unidad titulada Los amores de mi vida, en su primera sesión, señalo que le ha faltado tiempo para cerrar la clase. Durante la quinta sesión la profesora expreso, en referencia a una actividad ... Tenien molta vergonya d'actuar en públic i dues persones no ho han fet. 
Tabla 4

Sesiones según las características del alumnado.

\begin{tabular}{|c|c|c|c|c|c|c|c|c|c|c|c|}
\hline Indicadores & $\begin{array}{c}1 \\
\text { (Totalmen } \\
\text { te } \\
\text { desacuerd } \\
\text { o) }\end{array}$ & 2 & 3 & 4 & 5 & 6 & 7 & 8 & 9 & $\begin{array}{c}10 \\
\text { (Totalmen } \\
\text { te } \\
\text { deacuerd } \\
\text { o) }\end{array}$ & $\begin{array}{l}\text { No } \\
\text { proce } \\
\text { de }\end{array}$ \\
\hline $\begin{array}{l}\text { nivell } \\
\text { acadèmic de } \\
\text { l'alumnat }\end{array}$ & 0 & 0 & $\begin{array}{c}1,3 \\
\%\end{array}$ & 0 & $\begin{array}{c}5,3 \\
\%\end{array}$ & $\begin{array}{c}6,6 \\
\%\end{array}$ & $\begin{array}{c}11,8 \\
\%\end{array}$ & $\begin{array}{c}18,4 \\
\%\end{array}$ & $\begin{array}{c}36,8 \\
\%\end{array}$ & $19,7 \%$ & 0 \\
\hline Els gustos & 0 & 0 & 0 & $\begin{array}{c}5,2 \\
\% \\
\end{array}$ & $\begin{array}{c}7,8 \\
\% \\
\end{array}$ & $13 \%$ & $\begin{array}{c}23,4 \\
\% \\
\end{array}$ & $\begin{array}{c}32,5 \\
\%\end{array}$ & $6,5 \%$ & $2,6 \%$ & $9 \%$ \\
\hline $\begin{array}{l}\text { Les } \\
\text { característiq } \\
\text { ues culturals } \\
\text { del grup } \\
\text { classe }\end{array}$ & 0 & 0 & 0 & $\begin{array}{c}1,3 \\
\%\end{array}$ & $\begin{array}{c}9,2 \\
\%\end{array}$ & $\begin{array}{c}14,5 \\
\%\end{array}$ & $\begin{array}{c}22,4 \\
\%\end{array}$ & $\begin{array}{c}36,8 \\
\%\end{array}$ & $\begin{array}{c}15,8 \\
\%\end{array}$ & 0 & 0 \\
\hline $\begin{array}{l}\text { Els hàbits de } \\
\text { treball }\end{array}$ & 0 & 0 & 0 & $\begin{array}{c}5,2 \\
\% \\
\end{array}$ & $\begin{array}{c}7,8 \\
\% \\
\end{array}$ & $13 \%$ & $\begin{array}{c}15,6 \\
\% \\
\end{array}$ & $\begin{array}{c}41,6 \\
\% \\
\end{array}$ & $11,7 \%$ & $2,6 \%$ & $2,6 \%$ \\
\hline $\begin{array}{l}\text { La } \\
\text { sensibilitat } \\
\text { respecte a } \\
\text { les qüestions } \\
\text { de gènere }\end{array}$ & $3,9 \%$ & $\begin{array}{c}2,6 \\
\%\end{array}$ & 0 & 0 & $\begin{array}{c}7,8 \\
\%\end{array}$ & $13 \%$ & $7,8 \%$ & $\begin{array}{c}45,5 \\
\%\end{array}$ & $9,1 \%$ & $7,8 \%$ & $2,6 \%$ \\
\hline $\begin{array}{l}\text { La seva } \\
\text { competència } \\
\text { digital prèvia }\end{array}$ & 0 & 0 & 0 & 0 & $\begin{array}{c}5,2 \\
\%\end{array}$ & $\begin{array}{c}6,5 \\
\%\end{array}$ & $2,6 \%$ & $\begin{array}{c}46,8 \\
\%\end{array}$ & $\begin{array}{c}19,5 \\
\%\end{array}$ & $14,3 \%$ & $5,2 \%$ \\
\hline
\end{tabular}

Otra docente que aplico Los amores de mi vida, frente a la actividad ¿la Publicidad mata? Señalo:

L'alumnat fa també una valoració molt positiva de les activitats d'aquesta sessió. Una tercera profesora que utilizo el mismo material, expreso ... El tipus d'activitat ha provocat una reacció diferent per part dels nois i de les noies, hi ha hagut un biaix de gènere. Ho han trobat una mica obvi.

Uno de los profesores encuestados, que aplico la unidad Atenas el origen de la democracia, valoró positivamente la sesión 4, señalando:

La meva valoració de la sessió i la utilitat del contingut de la sessió són bones per l'adaptació que vaig fer dels materials del dossier pels meus alumnes perquè al grup en el que estic experimentant la unitat (i als altres dos grups de primer) els resultava molt difícil treballar el text de la Aristòtil de "La constitució d'Atenes" per la complexitat del contingut. També considero que les activitats de durant i després de la lectura són la majoria bastant complicades. A més no vam treballar les fonts 5, 6 i 7 perquè el ritme del meu grup és molt lent i necessitem avançar.

La docente que aplico The Maze: Nosotros y Ellos, da a conocer la opinión de los estudiantes en la primera sesión:

Els estudiants pensen que es tracta d'un vídeo joc. Estan més que engrescats. Els informo que es tracta de reflexionar i aprendre i que el classcraft és un mitja per obtenir puntuacions de forma lúdica. Segueixen molt engrescats, ho expliquen per tot arreu, els estudiants tenen cura del seu quadern, són competitius i ja anuncien qui guanyarà, de fet, tots els equips ja es senten guanyadors. D'altres estudiants que no tinc, m'aturen i em pregunten quan aniré a la seva aula. 


\section{Discusión y conclusiones}

Los materiales didácticos presentados, permiten que el alumnado amplíe su percepción sobre los temas tratados, mediante diversas estrategias, ejemplo, la indagación, la gamificación, etc. Las actividades propuestas dan la oportunidad al alumnado de resolver temas controversiales, como proponen Magendzo y Toledo (2015).

- Da la oportunidad de reflexión en el aula, el alumnado junto al profesor debate en torno a una temática particular, como señala Middaugh y Kahne, (2014).

- Promueve la incorporación de nuevos tratamientos didácticos, que incentivan competencia social y ciudadana, desde un enfoque maximalista (Kerr, 2002).

- Amplía los contenidos, permitiendo al alumnado analizar, juzgar e interpretar los hechos y sus consecuencias desde diferentes puntos de vista, rompiendo con el enfoque minimalista, que describe Kerr, (2002).

Desde el punto de vista de la construcción e implementación del material didáctico, según los docentes, es importante destacar:

- Que es un proceso lento, donde los estudiantes donde el alumnado debe solucionar problemas, analizando diversas fuentes de información. Las actividades que se desarrollan, dependerán de la postura de los docentes frente a la enseñanza de la ciudadanía, como explica Castillo (2016).

- El material se debe ir ajustando según las necesidades y habilidades específicas del alumnado, como señala Salazar-Jiménez (2016).

En conclusión, las actividades de las propuestas didácticas, según los/as docentes, favorecen el desarrollo de la competencia social y ciudadana, donde el alumno/a alcanza un rol central en el proceso de enseñanza-aprendizaje, reforzando habilidades de indagación y análisis. Por todo lo señalado, es necesario indicar que las propuestas didácticas realizadas han ofrecido la oportunidad de simular métodos de investigación propios del área de Historia y Ciencias Sociales, proporcionando un modelo que pueden ser aprovechados.

\section{Referencias}

Barriga-Ubed, E., y Salazar-Jiménez, R., (2015) Jóvenes y desafección política. Desafía ciudadano, una tarea de responsabilidad. VI Simposio Internacional Historia, patrimonio y educación cívica. Secretaría de Educación del Gobierno del Estado de Yucatán. Mérida (Yucatán, México).

Barriga-Ubed, E., Wilson-Daily, A. E., \& Prats-Cuevas, J. (2017). Aprender a votar con las elecciones al Parlamento Europeo. Una simulación innovadora del proceso electoral diseñado para el alumnado de Educación Secundaria Obligatoria, REIRE, Revista d'Innovació i Recerca en Educació, 10, (1).

Bisquerra, R. y Pérez-Escoda, N. (2015). ¿Pueden las escalas Likert aumentar en sensibilidad? REIRE, Revista d'Innovació i Recerca en Educació , 8 (2), 129-147. DOI: 10.1344/reire2015.8.2.828

Castillo, J. (2016) Formar en ciudadanía en el Chile actual. Una mirada a partir del desarrollo humano. Docencia, 58, 18-30.

Cerda, A. M., Loreto, M., Magendzo, A., Santa, E. y Varas, R. (2004). El complejo camino de la formación ciudadana. Una mirada a las prácticas docentes. Santiago: Lom ediciones. 
Gimeno, J. (coord.) (2009) Educar por competencias, ¿qué hay de nuevo? Madrid: Editorial Morata.

González-Valencia, G. y Santisteban-Fernández, A. (2016) La formación ciudadana en Colombia: entre la tradición y la transformación. Educación y Educadores., 19 (1), 89-102. DOI: 10.5294/edu.2016.19.1.5.

Kerr, D. (2002) An international review of citizenship in the curriculum: the IEA national case studies and the INCA archive. En G. Steiner-Khamsi, J. V. Torney-Purta y J. Schwille (Eds.), New Paradigms and Recurring Paradoxes in Education for Citizenship: An International Comparison (pp. 207-237). Amsterdam: Elsevier.

Magendzo, A. y Toledo, M. I. (2015). Educación en derechos humanos: Estrategia pedagógica-didáctica centrada en la controversia. Revista Electrónica Educare, 19(3), 116. doi: http://dx.doi.org/10.15359/ree.19-3.2

Middaugh, E., \& Kahne, J. (2014). Civic Development in Context. Educating Democratic Citizens in Troubled Times: Qualitative Studies of Current Efforts, 157.

Molina Neira, J. (2016). La Educación para la Ciudadanía Democrática en las aulas de Educación Secundaria Obligatoria: configuración de las ideas y actitudes sociopolíticas e interculturales del alumnado de Cataluña. Universidad de Barcelona. Tesis doctoral [disponible en: http://www.tdx.cat/handle/10803/399412 ]

Molina-Neira, J. (2017). Formar una ciudadanía crítica desde la enseñanza y el aprendizaje de la Historia: método, estrategias y contenidos clave. En Miralles, P., Gómez. C., Rodríguez, R. (Coord.) La enseñanza de la historia en el siglo XXI: Desarrollo y evaluación de competencias históricas para una ciudadanía democrática. (pp. 77-94). Murcia: EDITIUM

Muñoz, C. y Torres, B. (2014) La formación ciudadana en la escuela. Problemas y desafíos. Revista Electrónica Educare, 18 (2), 233-245. DOI:dx.doi.org/10.15359/ree.18-2.12

Orellana, Bivort, Martinez, Farias, Cárcamo, Blanco \& Perez (2015). Formación ciudadana y participación política en jóvenes de la Universidad del Bío-Bío, Chile. Civilizar, 119 - 134.

Prats, J., Fuentes, C., y Sabariego, M. (2019), Investigación evaluativa de materiales didácticos para la educación política y ciudadana a través de contenidos históricos. Revista Electrónica Interuniversitaria de Formación del Profesorado, 22(2), 1-14.

Prats, J. (1997) La selección de contenidos históricos para la educación secundaria. Iber: Didáctica de las ciencias sociales, geografía e historia, (12), 7-18.

Prats, J., y Santacana, J. (2011) Enseñar a pensar históricamente: la clase como simulación de la investigación histórica. En J. Prats (coord.) Didáctica de la Geografía y la Historia. Pp. 67-87. Barcelona: Graó,

Prats, J., Molina-Neira, J., Ruíz, A. \& Molina-Luque, F. (2017). Análisis de las Representaciones e Ideas Sociales del Alumnado en Educación para la Ciudadanía Democrática: Ejemplo de Mixed-methology desde y para la Investigación Transdisciplinar. International Journal of Sociology of Education, 6(1), 1-25.

Prats, J., y Wilson, A. (2013). ¿Aprender a votar? Una experiencia de educación política para alumnos de la ESO. IBER. Didáctica de las ciencias sociales, geografía e historia, 75.

Redón, S. (2010) La escuela como espacio de ciudadanía. Estudios Pedagógicos, 36 (2), 213239. 
Reyes, L., Campos, J., Osandón, L., y Muñoz, C. (2013) El profesorado y su rol en la formación de nuevos ciudadanos: desfases entre las comprensiones, las actuaciones y las expectativas. Estudios Pedagógicos, 39 (1), 217-237.

Salazar-Jiménez, R., Barriga-Ubed, E., y Ametller-López, Á. (2015) El aula como laboratorio de análisis histórico en 40 de ESO: El nacimiento del Fascismo en Europa. REIRE. Revista d'Innovació i Recerca en Educació, 8 (2), 94-115.

Salazar Jiménez, R. A. (2016) La construcción del discurso histórico en estudiantes de ESO: el aula como laboratorio. Universidad de Barcelona. Tesis doctoral [disponible en: http://www.tdx.cat/handle/10803/390947]

Salazar-Jiménez, R., Orellana-Fonseca, C., Muñoz-Labraña, C., \& Bellatti, I. (2017). El aula como laboratorio histórico: la guerra civil española. In Enseñanza de la historia y competencias educativas (pp. 123-140). Graó.

Toledo, M., Magendzo, A., Gutiérrez, V., \& Iglesias, R. (2015). Enseñanza de'temas controversiales' en la asignatura de historia y ciencias sociales desde la perspectiva de los profesores. Estudios pedagógicos (Valdivia), 41(1), 275-292. 